\title{
The Impact of Transposable Elements in Genome Evolution and Genetic Instability and Their Implications in Various Diseases
}

\author{
Selvam Ayarpadikannan, Heui-Soo Kim* \\ Department of Biological Sciences, College of Natural Sciences, Pusan National University, Busan 609-735, Korea
}

\begin{abstract}
Approximately $45 \%$ of the human genome is comprised of transposable elements (TEs). Results from the Human Genome Project have emphasized the biological importance of TEs. Many studies have revealed that TEs are not simply "junk" DNA, but rather, they play various roles in processes, including genome evolution, gene expression regulation, genetic instability, and cancer disposition. The effects of TE insertion in the genome varies from negligible to disease conditions. For the past two decades, many studies have shown that TEs are the causative factors of various genetic disorders and cancer. TEs are a subject of interest worldwide, not only in terms of their clinical aspects but also in basic research, such as evolutionary tracking. Although active TEs contribute to genetic instability and disease states, non-long terminal repeat transposons are well studied, and their roles in these processes have been confirmed. In this review, we will give an overview of the importance of TEs in studying genome evolution and genetic instability, and we suggest that further in-depth studies on the mechanisms related to these phenomena will be useful for both evolutionary tracking and clinical diagnostics.
\end{abstract}

Keywords: cancer, evolution, gene expression, transposable elements

\section{Introduction}

Transposable elements (TEs) are DNA sequences that are capable of integrating into the genome at a new site within the cell of its origin. Sometimes, the change in their positions creates or reverses mutations, thereby altering the cell's genotype. Barbara McClintock's discovery of these "jumping genes" earned her a Nobel Prize in 1983 [1]. TEs are prevalent in all plants and animals. In mammals, TEs and their remnants make up almost half of the genome, and in some plants, they constitute up to $90 \%$ of the genome [2]. TEs consist of two major classes: DNA transposons and retrotransposons. DNA transposons are capable of moving and inserting into new genomic sites [3]. Although they are currently not mobile in the human genome, they were active during early primate evolution until $\sim 37$ million years ago (Mya) [4]. Retrotransposons replicate by forming RNA intermediates, which are then reverse-transcribed to make DNA sequences and inserted into new genomic locations [4]. Based on the presence of long terminal repeats (LTRs), retrotransposons are further classified into two groups: LTR and non-LTR transposons. In humans, LTR elements are called human endogenous retroviruses (HERVs). It is estimated that HERVs inserted into the human genome $>25$ Mya $[5,6]$. Non-LTR retrotransposons include long interspersed element 1 (LINE-1 or L1), Alu, and SVA elements. Studies have revealed that these are the only TEs that are currently active in humans [7-11].

TEs have driven genome evolution in multiple ways. Retrotransposons comprise a large proportion of the genome, especially in plants and mammals. The effect of the increase in retrotransposons has been tolerated during evolution. Accumulating literature has proven that mobile elements are useful tools for studying genome evolution and gene function [12]. A comparative analysis has shown that the human genome makes 655 perfect full-length matches with vertebrate TEs. TE insertions have been shown to have many effects, such as regulation of gene expression, increased recombination rate, and unequal crossover. TE insertions have caused many effective changes in the human 
genome, and the selected changes have been responsible for the evolution of the human lineage [13]. The human genome contains many recently inserted active TEs, such as AluYa5, AluYb8, and AluYc1. Alu elements are a family of primatespecific short interspersed DNA elements. Various studies have proposed that Alu element insertions have created many variants that can potentially be used as DNA markers in human population studies, as well as in forensic analyses. Kass et al. [14] have identified an Alu-based polymorphism that consists of four alleles, from which the evolutionary order can be predicted.

The effects of TEs on genetic instability and human diseases have not been thoroughly studied. The mobile property of TEs is the reason for their mutational potential. TE insertions may create a broad range of effects on humans, ranging from silent mutations to alternative splicing. Both insertions and excisions of TEs can cause genomic instability, thus causing many human diseases, including genetic disorders, psychiatric problems, and cancer [15-21]. Furthermore, TE insertions may result in insertional mutations, non-allelic homologous recombination (NAHR), creation of novel regulatory sequences, and epigenetic changes [22]. A large number of human diseases that are associated with NAHR between Alu elements have been reported [22] Technical advances have helped to detect TE-associated diseases and develop novel biomarkers for clinical diagnostics. Computational tools have been developed to study the dynamics of transposition at a population level, thus providing critical insights into the mechanisms behind genome evolution. Finally, combining the genomic materials from diverse individuals followed by high-throughput sequencing can enhance the significance of characterizing genomic polymorphisms in a population [23].

In this review, we will provide updates on our current understanding of the roles of TEs in genome evolution and genetic instability. Further, we focus on how their activity affects gene expression and causes disease states in human beings.

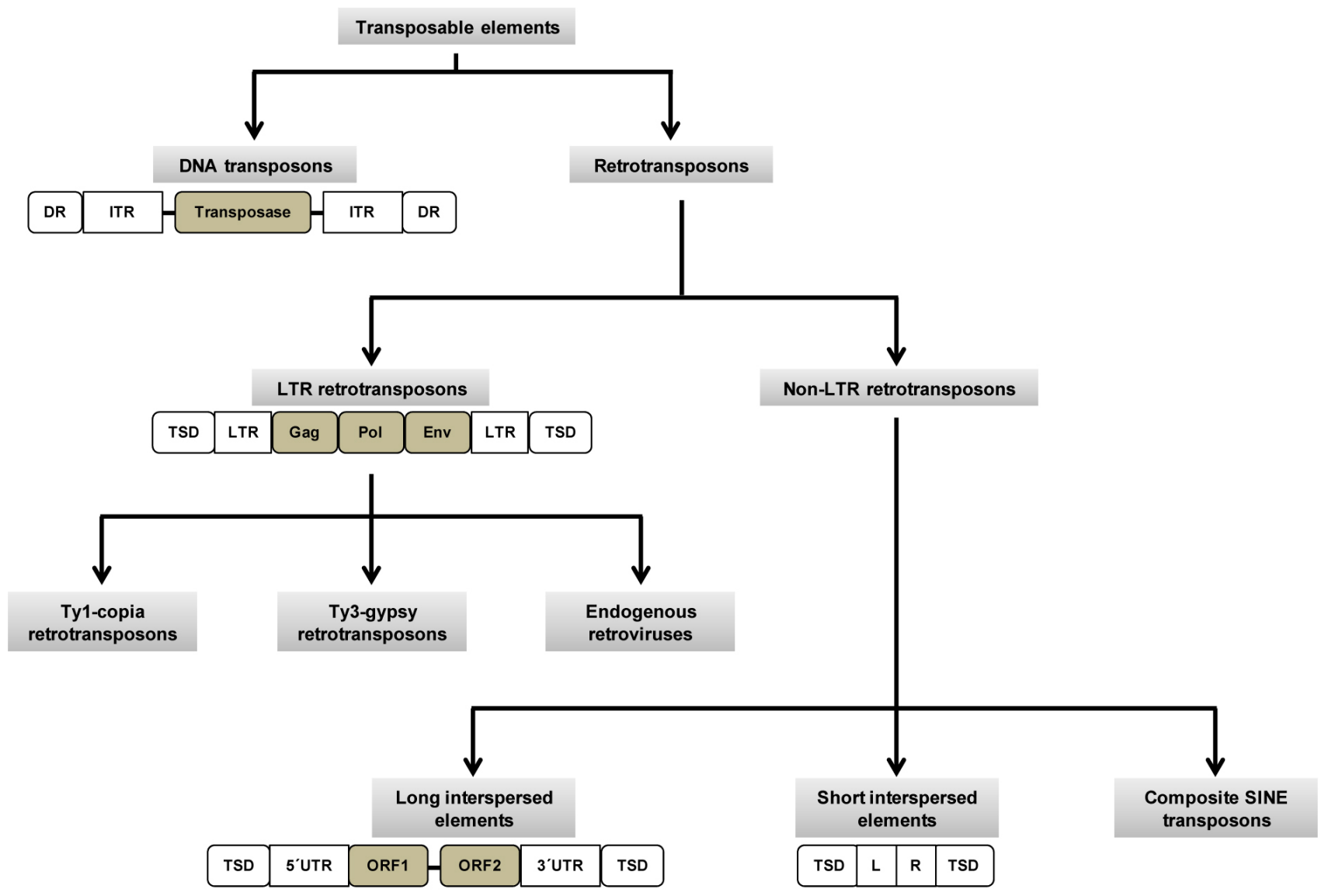

Fig. 1. Classification and structure of transposable elements. DRs, direct repeats; ITRs, inverted terminal repeats; TSD, tandem site duplication; LTRs, long terminal repeats; UTRs, untranslated region; ORF, open reading frame; L, left; R, right; SINE, short interspersed nuclear element. 


\section{Role of Retrotransposons in Human Genome Evolution}

Brain evolution is an important process that accelerated the evolution of humans. This occurred due to natural selection and genomic variation, a major source of which has been TE insertions. TE insertions contributed markedly to variation and increased the speed of evolution [24-26]. Furthermore, they increase the recombination rate, in addition to affecting genes and their expression [24, 26]. From an evolutionary perspective, humans are unique in the speed of evolution and the number and activity of TEs. It is proposed that the high frequency of TE insertions is responsible at least in part for the rapid evolution of humans. It is speculated that some sets of genes might have been activated or suppressed due to variations caused by TE insertions, which in turn increased the chances for evolution [13]. There has been a prominent impact of retrotransposons on human evolution at the genomic level. Retrotransposons have shaped human evolution at the RNA level through various mechanisms, such as modulation of gene expression, RNA editing, and epigenetic regulation [27].

\section{LTR retrotransposons}

In humans, LTR retrotransposons are called HERVs (Fig. 1 ), which constitute $5 \%$ of the genome. The human genome shows $99 \%$ similarity with chimpanzees and bonobos. Hence, the differences between these species are likely to be in regulatory sequences: promoters, enhancers, polyadenylation signals, and transcription factor (TF) binding sites. The LTRs of HERVs help in regulating the expression of nearby genes. The active human-specific LTRs that have been identified belong to the HERV-K family. It is proposed that some of these endogenous retroviruses may have integrated into regulatory regions of the human genome and that they eventually contributed to human evolution [28]. Khodosevich et al. [28] suggested that regulatory sequences found in retroviral LTRs may alter the expression of (or even inactivate) adjacent genes. On the other hand, HERV insertion may benefit the host, for example, by reverting harmful mutation [28].

\section{Non-LTR retrotransposon}

Human non-LTR retroposons include both active (L1, Alu, and SVA) and inactive elements (L2 and mammalian-wide interspersed repeat). Although more than 500,000 copies of L1 elements are found in the human genome, only 100 copies are known to be intact [29]. An intact L1 element is approximately 6 kilobases $(\mathrm{kb})$ in length, with a $5^{\prime}$ untranslated region (UTR) containing an internal RNA polymerase II promoter, two open reading frames (ORF1 and
ORF2), and a 3' UTR (Fig. 1) [29, 30]. L1 elements are the only autonomous TEs in the human genome because of their retrotransposition property, called target-primed reverse transcription [27]. Alu elements are often called "a parasite's parasite," because they do not code for a polymerase and, hence, are non-autonomous in nature. Alu elements depend on L1 elements for retrotransposition machinery [31-33]. However, they are considered the most successful TEs in the human genome in terms of copy number [27]. An intact SVA element is approximately $2 \mathrm{~kb}$ in length, which includes a hexamer repeat, an Alu-like region, a variable number of tandem repeats, and a HERV-K10-like sequence. SVA elements are also non-autonomous and most likely depend on L1 retrotransposition machinery [34, 35].

Two key features of non-LTR retrotransposons that control retrotransposition activity are high copy number and continued activity over millions of years [5, 27]. From an evolutionary perspective, the observed uniqueness of nonLTR retrotransposons is due to their vertical transfer in both primates and mammals [4, 5, 36]. Amplification rates among non-LTR retrotransposons are not uniform (Alu, 40 Mya; L1, 12-40 Mya; SVA, 6 Mya) [27]. Among non-LTR retrotransposons, Alu elements are the most thoroughly studied in relation to evolution. Alu repeats may cause genomic diversity in various ways. Their amplification has enabled them to become the largest family of mobile elements in the human genome. It is estimated that thousands of Alu elements have integrated into the human genome since the divergence of humans and African apes [37-40]. Although some Alu insertions have caused harmful mutations, most have contributed to genetic diversity [8]. Moreover, Alu repeats have also influenced the accumulation of single-nucleotide polymorphisms in the genome [30, 40, 41]. From previous reports, it is evident that most of the recent Alu insertions are the source of genetic variations, which have been useful for studying both the relationships between populations and the evolution and organization of tandem-arrayed gene families [37-40]. Batzer and Deninger [42] suggested that the Alu insertion, in relation to genetic variation, may also be useful in generating species-specific genetic markers.

\section{Roles of TEs in Genomic Instability and Dis- ease States}

TEs can cause genomic instability either by insertions or by rearrangements in the genome. Notably, structural variations in the human genome are the primary cause of inter-individual variability. Structural variations include insertion, deletion, inversion, duplication, and translocation. The characteristics of TEs, such as abundance in the 
genome, high sequence identity, and ability to move, make them major contributors to genomic instability [5, 27, 43]. Recent studies have revealed the implications of TEs in genomic instability and human genome evolution [44]. Mutations associated with TE insertions are well studied, and approximately $0.3 \%$ of all mutations are caused by retrotransposon insertions [27]. Such insertions can be deleterious by disrupting the regulatory sequences of a gene. When a TE inserts within an exon, it may change the ORF, such that it codes for an aberrant peptide, or it may even cause missense or nonsense mutations. On the other hand, if it is inserted into an intronic region, it may cause an alternative splicing event by introducing novel splice sites, disrupting the canonical splice site, or introducing a polyadenylation signal [8-11, 42, 43]. In some instances, TE insertion into intronic regions can cause mRNA destabilization, thereby reducing gene expression [45]. Similarly, some studies have suggested that TE insertion into the $5^{\prime}$ or $3^{\prime}$ region of a gene may alter its expression [46-48]. Thus, such a change in gene expression may, in turn, change the equilibrium of regulatory networks and result in disease conditions (reviewed in Konkel and Batzer [43]).

The currently active non-LTR transposons, L1, SVA, and

Table 1. Disease conditions associated with transposable element (TE) insertions

\begin{tabular}{|c|c|c|}
\hline Gene & Type of TE & Disease/genetic disorder \\
\hline$A P C$ & Alu & Colon cancer [63] \\
\hline BRCA1 & Alu & Breast cancer/ovarian cancer [48] \\
\hline$B R C A 2$ & Alu & Breast cancer/ovarian cancer [17] \\
\hline MLVI2 & Alu & Leukemia $[64]$ \\
\hline NF1 & Alu & Neurofibromatosis type I [65] \\
\hline$A P C$ & Alu & Cancer of the colon $[8,20]$ \\
\hline$B A A T$ & LTR & Hypercholanemia [66] \\
\hline$M S L N$ & LTR & Cancer [67] \\
\hline$A D H 1 C$ & LTR & Role in alcoholism [68] \\
\hline HSD17B1 & LTR & Breast cancer $[69]$ \\
\hline FKTN & L1 & $\begin{array}{l}\text { Fukuyama-type congenital muscular } \\
\text { dystrophy }[70,71]\end{array}$ \\
\hline$D M D$ & L1 & Duchenne muscular dystrophy [71] \\
\hline$C Y B B$ & L1 & Chronic granulomatous disease [72] \\
\hline$R P 2$ & $\mathrm{~L} 1$ & X-linked retinitis pigmentosa [73] \\
\hline F8 & Alu & Hemophilia A [74] \\
\hline$C Y B B$ & L1 & Chronic granulomatous disease [72] \\
\hline U2AF65 & Alu & $\begin{array}{l}\text { Loss of hnRNP C binding, leading to } \\
\text { aberrant exonization [75] }\end{array}$ \\
\hline OAT & Alu & OAT deficiency [76] \\
\hline COL4A3 & Alu & Alport syndrome [77] \\
\hline GUSB & Alu & Sly syndrome [78] \\
\hline PDHX & L1 & $\begin{array}{l}\text { Pyruvate dehydrogenase complex } \\
\text { deficiency [79] }\end{array}$ \\
\hline RPS6KA3 & L1 & Coffin-Lowry syndrome [80] \\
\hline
\end{tabular}

LTR, long terminal repeat.
$A l u$, are reported to be the causative factors of many genetic disorders, such as hemophilia, Apert syndrome, familial hypercholesterolemia, and colon and breast cancer (Table 1) $[8,10,11,27]$. Among the reported TE-mediated genetic disorders, X-linked diseases are more abundant than autosomal diseases [11,27, 45], most of which are caused by L1 insertions. However, the phenomenon behind L1 and $\mathrm{X}$-linked genetic disorders has not yet been revealed. The breast cancer 2 (BRCA2) gene, associated with breast and ovarian cancers, has been reported to be disrupted by multiple non-LTR TE insertions [9, 18, 49]. There are some reports that the same location of a gene may undergo multiple insertions (e.g., Alu and L1 insertions in the adenomatous polyposis coli gene) (Table 1).

It has also been proposed that inverted repeats are likely to be hotspots of genomic instability [50]. Closely occurring Alu repeats form hairpin structures that are prone to double-strand breaks (DSBs) and excision [50, 51]. In addition, de novo Alu insertions may create new inverted repeats that result in rearrangements in future generations [43]. Due to the abundance of TEs in the human genome, the probability of TE-mediated NAHR translocations is high. Kolomietz et al. [52] reported that Alu elements are often found in and around the breakage points of translocations and result in diseases [53]. Some studies have analyzed the human genome using the chimpanzee reference genome and found that deletions that are caused by Alu-mediated NAHRs are 9 times more frequent than L1-mediated NAHRs in the human genome [54, 55]. Alu-mediated NAHRs are known to be associated with various genetic disorders and cancer (Table 1) $[8,10]$. L1 endonuclease creates abundant DSBs that are required for retrotransposition in mammalian cells and eventually contribute to genomic instability [56]. However, it has been difficult to define this in an experimental condition, as physiological conditions cannot be simulated in vitro. Moreover, it is also difficult to distinguish DSBs caused by L1 from DSBs caused by other mechanisms in vivo [44, 56].

From recent studies, it is apparent that the methylation state of DNA is associated with cancer [57]. TEs - in particular, the promoters of $\mathrm{L} 1$ elements - are reported to be demethylated in cancer cells $[58,59]$. On the other hand, the methylation of retrotransposons is supposed to be a defense mechanism against retrotransposition in somatic cells [60]. It has also been reported by some studies that the L1 transcription rate is increased in hypomethylated cancer cells $[58,61]$. Demethylation of TE promoters may result in their activation, which in turn could modify the TF level in the cell. It is possible that such changes in TF levels lead to alterations in global gene expression [57]. In addition, demethylation may result in the activation of the L1 
antisense promoter, which may eventually produce cancerassociated chimeric transcripts [62, 63].

\section{Conclusion and Future Perspectives}

Taken together, the mechanisms discussed above have demonstrated the considerable impact of TEs on human genome evolution, genetic instability, and disease occurrence. There has been a recent increase in studies demonstrating the roles of TEs in multiple molecular processes. Importantly, several studies have found an association between TEs and cancer conditions. Technological developments have led to promising techniques (e.g., next-generation sequencing) that will assist researchers in studying, understanding, and confirming the role of TEs in genetic instability and diseases. Such progress may lead to the development of novel therapeutic strategies in the near future, such as personalized gene therapy for the treatment of genetic disorders. The clinical community has already realized the importance of personalized cancer treatments and is moving toward excellence in such treatment strategies. Therefore, we believe that in-depth studies on the role(s) of TEs in evolution, the epigenetic control of gene expression, and clinical aspects will be of paramount importance in uncovering novel mechanisms that can be targeted for therapeutic intervention.

\section{Acknowledgments}

This research was supported by awards from the AGENDA project (Project No. PJ009254) in the National Institute of Animal Science, Rural Development Administration (RDA).

\section{References}

1. McClintock B. CB. The origin and behavior of mutable loci in maize. Proc Natl Acad Sci U S A 1950;36:344-355.

2. SanMiguel P, Tikhonov A, Jin YK, Motchoulskaia N, Zakharov D, Melake-Berhan A, et al. Nested retrotransposons in the intergenic regions of the maize genome. Science 1996;274:765768.

3. Craig NL, Craigie R, Gellert M, Lambowitz AM. Mobile DNA II. Washington, DC: ASM Press, 2002.

4. Pace JK 2nd, Feschotte C. The evolutionary history of human DNA transposons: evidence for intense activity in the primate lineage. Genome Res 2007; 17:422-432.

5. Lander ES, Linton LM, Birren B, Nusbaum C, Zody MC, Baldwin J, et al. Initial sequencing and analysis of the human genome. Nature 2001;409:860-921.

6. Mills RE, Bennett EA, Iskow RC, Devine SE. Which transposable elements are active in the human genome? Trends Genet 2007;23:183-191.
7. Kazazian HH Jr, Wong C, Youssoufian H, Scott AF, Phillips DG, Antonarakis SE. Haemophilia A resulting from de novo insertion of $\mathrm{L} 1$ sequences represents a novel mechanism for mutation in man. Nature 1988;332:164-166.

8. Deininger PL, Batzer MA. Alu repeats and human disease. Mol Genet Metab 1999;67:183-193.

9. Chen JM, Stenson PD, Cooper DN, Férec C. A systematic analysis of LINE-1 endonuclease-dependent retrotranspositional events causing human genetic disease. Hum Genet 2005;117: 411-427.

10. Callinan PA, Batzer MA. Retrotransposable elements and human disease. Genome Dyn 2006;1:104-115.

11. Belancio VP, Hedges DJ, Deininger P. Mammalian non-LTR retrotransposons: for better or worse, in sickness and in health. Genome Res 2008;18:343-358.

12. Kazazian HH Jr. Mobile elements: drivers of genome evolution. Science 2004;303:1626-1632.

13. Britten RJ. Transposable element insertions have strongly affected human evolution. Proc Natl Acad Sci U S A 2010;107: 19945-19948.

14. Kass DH, Jamison N, Mayberry MM, Tecle E. Identification of a unique Alu-based polymorphism and its use in human population studies. Gene 2007;390:146-152.

15. Tighe PJ, Stevens SE, Dempsey S, Le Deist F, Rieux-Laucat F, Edgar JD. Inactivation of the Fas gene by Alu insertion: retrotransposition in an intron causing splicing variation and autoimmune lymphoproliferative syndrome. Genes Immun 2002;3 Suppl 1:S66-S70.

16. Mukherjee S, Mukhopadhyay A, Banerjee D, Chandak GR, Ray K. Molecular pathology of haemophilia B: identification of five novel mutations including a LINE 1 insertion in Indian patients. Haemophilia 2004;10:259-263.

17. Miki Y, Nishisho I, Horii A, Miyoshi Y, Utsunomiya J, Kinzler $\mathrm{KW}$, et al. Disruption of the APC gene by a retrotransposal insertion of L1 sequence in a colon cancer. Cancer Res 1992;52: 643-645.

18. Miki Y, Katagiri T, Kasumi F, Yoshimoto T, Nakamura Y. Mutation analysis in the BRCA2 gene in primary breast cancers. Nat Genet 1996;13:245-247.

19. Johnston JB, Silva C, Holden J, Warren KG, Clark AW, Power C. Monocyte activation and differentiation augment human endogenous retrovirus expression: implications for inflammatory brain diseases. Ann Neurol 2001;50:434-442.

20. Wallace MR, Andersen LB, Saulino AM, Gregory PE, Glover TW, Collins FS. A de novo Alu insertion results in neurofibromatosis type 1. Nature 1991;353:864-866.

21. Guffanti G, Gaudi S, Fallon JH, Sobell J, Potkin SG, Pato C, et al. Transposable elements and psychiatric disorders. Am J Med Genet B Neuropsychiatr Genet 2014;165B:201-216.

22. Belancio VP, Deininger PL, Roy-Engel AM. LINE dancing in the human genome: transposable elements and disease. Genome Med 2009;1:97.

23. Zhuang J, Wang J, Theurkauf W, Weng Z. TEMP: a computational method for analyzing transposable element polymorphism in populations. Nucleic Acids Res 2014;42:68266838.

24. Jurka J. Repbase update: a database and an electronic journal 
of repetitive elements. Trends Genet 2000;16:418-420.

25. Mills RE, Bennett EA, Iskow RC, Luttig CT, Tsui C, Pittard WS, et al. Recently mobilized transposons in the human and chimpanzee genomes. Am J Hum Genet 2006;78:671-679.

26. Ponicsan SL, Kugel JF, Goodrich JA. Genomic gems: SINE RNAs regulate mRNA production. Curr Opin Genet Dev 2010; 20:149-155.

27. Cordaux R, Batzer MA. The impact of retrotransposons on human genome evolution. Nat Rev Genet 2009;10:691-703.

28. Khodosevich K, Lebedev Y, Sverdlov E. Endogenous retroviruses and human evolution. Comp Funct Genomics 2002;3: 494-498.

29. Brouha B, Schustak J, Badge RM, Lutz-Prigge S, Farley AH, Moran JV, et al. Hot L1s account for the bulk of retrotransposition in the human population. Proc Natl Acad Sci U S A 2003;100:5280-5285.

30. Swergold GD. Identification, characterization, and cell specificity of a human LINE-1 promoter. Mol Cell Biol 1990;10:67186729.

31. Babushok DV, Kazazian HH Jr. Progress in understanding the biology of the human mutagen LINE-1. Hum Mutat 2007;28: 527-539.

32. Dewannieux M, Esnault C, Heidmann T. LINE-mediated retrotransposition of marked Alu sequences. Nat Genet 2003;35: 41-48.

33. Weiner AM. SINEs and LINEs: the art of biting the hand that feeds you. Curr Opin Cell Biol 2002;14:343-350.

34. Ostertag EM, Goodier JL, Zhang Y, Kazazian HH Jr. SVA elements are nonautonomous retrotransposons that cause disease in humans. Am J Hum Genet 2003;73:1444-1451.

35. Wang H, Xing J, Grover D, Hedges DJ, Han K, Walker JA, et al. SVA elements: a hominid-specific retroposon family. J Mol Biol 2005;354:994-1007.

36. Cantrell MA, Scott L, Brown CJ, Martinez AR, Wichman HA. Loss of LINE-1 activity in the megabats. Genetics 2008;178: 393-404

37. Carroll ML, Roy-Engel AM, Nguyen SV, Salem AH, Vogel E, Vincent B, et al. Large-scale analysis of the Alu Ya5 and Yb8 subfamilies and their contribution to human genomic diversity. J Mol Biol 2001;311:17-40.

38. Roy-Engel AM, Carroll ML, Vogel E, Garber RK, Nguyen SV, Salem AH, et al. Alu insertion polymorphisms for the study of human genomic diversity. Genetics 2001;159:279-290.

39. Roy-Engel AM, Carroll ML, El-Sawy M, Salem AH, Garber RK, Nguyen SV, et al. Non-traditional Alu evolution and primate genomic diversity. J Mol Biol 2002;316:1033-1040.

40. Roy AM, Carroll ML, Nguyen SV, Salem AH, Oldridge M, Wilkie AO, et al. Potential gene conversion and source genes for recently integrated Alu elements. Genome Res 2000;10: 1485-1495.

41. Roy AM, Carroll ML, Kass DH, Nguyen SV, Salem AH, Batzer MA, et al. Recently integrated human Alu repeats: finding needles in the haystack. Genetica 1999;107:149-161.

42. Batzer MA, Deininger PL. Alu repeats and human genomic diversity. Nat Rev Genet 2002;3:370-379.

43. Konkel MK, Batzer MA. A mobile threat to genome stability: the impact of non-LTR retrotransposons upon the human genome. Semin Cancer Biol 2010;20:211-221.

44. Hedges DJ, Deininger PL. Inviting instability: transposable elements, double-strand breaks, and the maintenance of genome integrity. Mutat Res 2007;616:46-59.

45. Chen JM, Férec C, Cooper DN. LINE-1 endonuclease-dependent retrotranspositional events causing human genetic disease: mutation detection bias and multiple mechanisms of target gene disruption. J Biomed Biotechnol 2006;2006:56182.

46. Sobczak K, Krzyzosiak WJ. Structural determinants of BRCA1 translational regulation. J Biol Chem 2002;277:17349-17358.

47. Landry JR, Medstrand P, Mager DL. Repetitive elements in the 5 ' untranslated region of a human zinc-finger gene modulate transcription and translation efficiency. Genomics 2001;76: 110-116.

48. Chen LL, Carmichael GG. Gene regulation by SINES and inosines: biological consequences of A-to-I editing of Alu element inverted repeats. Cell Cycle 2008;7:3294-3301.

49. Teugels E, De Brakeleer S, Goelen G, Lissens W, Sermijn E, De Grève J. De novo Alu element insertions targeted to a sequence common to the BRCA1 and BRCA2 genes. Hum Mutat 2005; $26: 284$.

50. Lobachev KS, Stenger JE, Kozyreva OG, Jurka J, Gordenin DA, Resnick MA. Inverted Alu repeats unstable in yeast are excluded from the human genome. EMBOJ 2000;19:3822-3830.

51. Voineagu I, Narayanan V, Lobachev KS, Mirkin SM. Replication stalling at unstable inverted repeats: interplay between DNA hairpins and fork stabilizing proteins. Proc Natl Acad Sci U S A 2008;105:9936-9941.

52. Kolomietz E, Meyn MS, Pandita A, Squire JA. The role of Alu repeat clusters as mediators of recurrent chromosomal aberrations in tumors. Genes Chromosomes Cancer 2002;35:97-112.

53. Onno M, Nakamura T, Hillova J, Hill M. Rearrangement of the human tre oncogene by homologous recombination between Alu repeats of nucleotide sequences from two different chromosomes. Oncogene 1992;7:2519-2523.

54. Sen SK, Han K, Wang J, Lee J, Wang H, Callinan PA, et al. Human genomic deletions mediated by recombination between Alu elements. Am J Hum Genet 2006;79:41-53.

55. Han K, Lee J, Meyer TJ, Remedios P, Goodwin L, Batzer MA. L1 recombination-associated deletions generate human genomic variation. Proc Natl Acad Sci U S A 2008;105:19366-19371.

56. Gasior SL, Wakeman TP, Xu B, Deininger PL. The human LINE-1 retrotransposon creates DNA double-strand breaks. J Mol Biol 2006;357:1383-1393.

57. Wilson AS, Power BE, Molloy PL. DNA hypomethylation and human diseases. Biochim Biophys Acta 2007;1775:138-162.

58. Daskalos A, Nikolaidis G, Xinarianos G, Savvari P, Cassidy A, Zakopoulou R, et al. Hypomethylation of retrotransposable elements correlates with genomic instability in non-small cell lung cancer. Int J Cancer 2009;124:81-87.

59. Suter CM, Martin DI, Ward RL. Hypomethylation of L1 retrotransposons in colorectal cancer and adjacent normal tissue. Int J Colorectal Dis 2004;19:95-101.

60. Yoder JA, Walsh CP, Bestor TH. Cytosine methylation and the ecology of intragenomic parasites. Trends Genet 1997;13:335340.

61. Florl AR, Löwer R, Schmitz-Dräger BJ, Schulz WA. DNA 
methylation and expression of LINE-1 and HERV-K provirus sequences in urothelial and renal cell carcinomas. Br J Cancer 1999;80:1312-1321

62. Speek M. Antisense promoter of human L1 retrotransposon drives transcription of adjacent cellular genes. Mol Cell Biol 2001;21:1973-1985.

63. Cruickshanks HA, Tufarelli C. Isolation of cancer-specific chimeric transcripts induced by hypomethylation of the LINE-1 antisense promoter. Genomics 2009;94:397-406.

64. Halling KC, Lazzaro CR, Honchel R, Bufill JA, Powell SM, Arndt CA, et al. Hereditary desmoid disease in a family with a germline Alu I repeat mutation of the APC gene. Hum Hered 1999;49:97-102.

65. Economou-Pachnis A, Tsichlis PN. Insertion of an Alu SINE in the human homologue of the Mlvi-2 locus. Nucleic Acids Res 1985;13:8379-8387.

66. Su LK, Steinbach G, Sawyer JC, Hindi M, Ward PA, Lynch PM. Genomic rearrangements of the APC tumor-suppressor gene in familial adenomatous polyposis. Hum Genet 2000;106:101107.

67. Carlton VE, Harris BZ, Puffenberger EG, Batta AK, Knisely AS, Robinson DL, et al. Complex inheritance of familial hypercholanemia with associated mutations in TJP2 and BAAT. Nat Genet 2003;34:91-96.

68. Hassan R, Ho M. Mesothelin targeted cancer immunotherapy. Eur J Cancer 2008;44:46-53.

69. Edenberg HJ. Regulation of the mammalian alcohol dehydrogenase genes. Prog Nucleic Acid Res Mol Biol 2000;64:295341.

70. Tsuchiya M, Nakao H, Katoh T, Sasaki H, Hiroshima M, Tanaka $\mathrm{T}$, et al. Association between endometriosis and genetic polymorphisms of the estradiol-synthesizing enzyme genes HSD17B1 and CYP19. Hum Reprod 2005;20:974-978.

71. Kondo-Iida E, Kobayashi K, Watanabe M, Sasaki J, Kumagai T, Koide $\mathrm{H}$, et al. Novel mutations and genotype-phenotype relationships in 107 families with Fukuyama-type congenital muscular dystrophy (FCMD). Hum Mol Genet 1999;8:23032309.
72. Narita N, Nishio H, Kitoh Y, Ishikawa Y, Ishikawa Y, Minami $\mathrm{R}$, et al. Insertion of a 5 ' truncated $\mathrm{L} 1$ element into the 3 ' end of exon 44 of the dystrophin gene resulted in skipping of the exon during splicing in a case of Duchenne muscular dystrophy. J Clin Invest 1993;91:1862-1867.

73. Meischl C, Boer M, Ahlin A, Roos D. A new exon created by intronic insertion of a rearranged LINE-1 element as the cause of chronic granulomatous disease. Eur J Hum Genet 2000;8: 697-703.

74. Schwahn U, Lenzner S, Dong J, Feil S, Hinzmann B, van Duijnhoven G, et al. Positional cloning of the gene for X-linked retinitis pigmentosa 2. Nat Genet 1998;19:327-332.

75. Ganguly A, Dunbar T, Chen P, Godmilow L, Ganguly T. Exon skipping caused by an intronic insertion of a young Alu $\mathrm{Yb} 9 \mathrm{el}-$ ement leads to severe hemophilia A. Hum Genet 2003;113:348352.

76. Zarnack K, König J, Tajnik M, Martincorena I, Eustermann S, Stévant I, et al. Direct competition between hnRNP C and U2AF65 protects the transcriptome from the exonization of Alu elements. Cell 2013;152:453-466.

77. Mitchell GA, Labuda D, Fontaine G, Saudubray JM, Bonnefont JP, Lyonnet $\mathrm{S}$, et al. Splice-mediated insertion of an Alu sequence inactivates ornithine delta-aminotransferase: a role for Alu elements in human mutation. Proc Natl Acad Sci U S A 1991;88:815-819.

78. Knebelmann B, Forestier L, Drouot L, Quinones S, Chuet C, Benessy F, et al. Splice-mediated insertion of an Alu sequence in the COL4A3 mRNA causing autosomal recessive Alport syndrome. Hum Mol Genet 1995;4:675-679.

79. Vervoort R, Gitzelmann R, Lissens W, Liebaers I. A mutation (IVS8+0.6kbdelTC) creating a new donor splice site activates a cryptic exon in an Alu-element in intron 8 of the human beta-glucuronidase gene. Hum Genet 1998;103:686-693.

80. Mine M, Chen JM, Brivet M, Desguerre I, Marchant D, de Lonlay $\mathrm{P}$, et al. A large genomic deletion in the PDHX gene caused by the retrotranspositional insertion of a full-length LINE-1 element. Hum Mutat 2007;28:137-142. 